\title{
LÂMINAS DE IRRIGAÇÃO E GENÓTIPOS NA PRODUÇÃO E QUALIDADE DE FRUTOS DE BANANEIRA ${ }^{1}$
}

\author{
Juracy Rocha Braga Filho², Jorge Luiz do Nascimento ${ }^{3}$, Ronaldo Veloso Naves 3 , \\ Maria Célia Lopes Torres ${ }^{3}$, Robson Maia Geraldine ${ }^{3}$, Eli Regina Barboza de Souza ${ }^{3}$, Flávio Vieira Barroso ${ }^{4}$
}

\section{ABSTRACT \\ IRRIGATION LEVELS AND GENOTYPES ON \\ BANANA TREE FRUITS QUALITY AND YIELD}

Brazil is the fourth biggest banana producer. However, its social function is more important than the economic one. In this study, the productive features of banana tree genotypes, under different irrigation levels, and the sensorial characteristic for taste of ripe fruits were evaluated. The experiment was carried out from January 2004 to July 2005, with the following genotypes: false FHIA 18, Grand Naine, Prata, and Thap Maeo. After resting for two days in a cold chamber, the fruits were evaluated in the third, fourth and fifth days, for determining the preferred sample. The four genotypes presented significant results to irrigation. The water levels effect was verified in the weight of the bunch, rachis, and hands, and in the total number of fruits, bunch length and number of hands. The weight of the bunch and hands, and the total number of fruits, in the false FHIA 18, Grand Naine, and Thap Maeo genotypes, presented similar responses, except for the weight of bunch and hands, in Thap Maeo. The effect of the water levels among the genotypes occurred on most variables, except for the weight and the number of fruits of the second hand and diameter of the central fruit. False FHIA 18 presented more variables influenced by the irrigation levels and, for Prata, the only influence occurred in the number of hands. The irrigation positively influenced the productive characteristics of the false FHIA 18, Grand Naine, and Thap Maeo banana trees, which showed to be promising for the Brazilian savannah region. Consumers prefer the Prata and Grand Naine genotypes.

KEY-WORDS: Musa sp.; Grand Naine; false FHIA 18; Thap Maeo.

\section{INTRODUÇÃO}

A Índia é o maior produtor de banana do mundo, seguida da China, Filipinas e do Brasil. Este, por sua vez, é o segundo produtor em área colhida, sendo inferior apenas à Índia, em 2007. Equador e Bélgica foram os maiores exportadores e

\section{RESUMO}

O Brasil ocupa a quarta posição no ranking dos países produtores de banana. Contudo, maior que sua importância econômica, é sua função social. Neste estudo, foram avaliadas as características produtivas de genótipos de bananeira, sob diferentes lâminas de irrigação, e a característica sensorial de sabor de frutos maduros. O experimento foi conduzido de janeiro de 2004 a julho de 2005, com os genótipos falsa FHIA 18, Grande Naine, Prata e Thap Maeo. Após dois dias de descanso em câmara fria, os frutos foram avaliados no terceiro, quarto e quinto dias, para determinação da amostra preferida. Os quatro genótipos apresentaram respostas significativas à irrigação. $\mathrm{O}$ efeito das lâminas foi verificado na massa do cacho, da ráquis e das pencas, e no número total de frutos, comprimento do cacho e número de pencas. A massa do cacho e das pencas e o número total de frutos, nos genótipos falsa FHIA 18, Grande Naine e Thap Maeo, apresentaram resposta semelhante, exceto para a massa do cacho e das pencas, em Thap Maeo. O efeito das lâminas dentro de genótipos ocorreu para as demais variáveis, exceto para a massa e o número de frutos da segunda penca e diâmetro do fruto central. Falsa FHIA 18 teve mais variáveis influenciadas pelas lâminas de irrigação, enquanto, para Prata, somente ocorreu influência na variável número de pencas. A irrigação influenciou positivamente as características produtivas das bananeiras falsa FHIA 18, Grande Naine e Thap Maeo, que se mostraram promissoras para a região do Cerrado. Os genótipos Prata e Grande Naine são os preferidos pelos consumidores.

PALAVRAS-CHAVE: Musa sp.; Grande Naine; Falsa FHIA 18; Thap Maeo.

importadores mundiais, respectivamente, em 2006 (Agrianual 2009). Existe, no Brasil, um número expressivo de variedades de bananeira, porém, poucas são utilizadas comercialmente, devido a aspectos como produtividade, tolerância a pragas, resistência à seca e ao frio, porte e preferência do consumidor (Alves 1999).

1. Trabalho recebido em mar./2010 e aceito para publicação em mar./2011 (nº registro: PAT 9041/ DOI: 10.5216/pat.v41i2.9041).

2. Agência Goiana de Defesa Agropecuária (Agrodefesa), Goiânia, GO, Brasil. E-mail: juracyr@yahoo.com.br.

3. Universidade Federal de Goiás, Escola de Agronomia e Engenharia de Alimentos, Goiânia, GO, Brasil.

E-mails: jln@agro.ufg.br, ronaldo@agro.ufg.br, eliregina1@gmail.com, celialopes@agro.ufg.br, robson.agro.ufg@gmail.com.

4. Engenheiro Agrônomo, Goiânia, GO, Brasil.E-mail: flaviovieirabarroso@yahoo.com.br. 
A banana faz parte da dieta do brasileiro e, maior que sua importância econômica, é sua função social, podendo ser encontrada desde em plantios comerciais até no cultivo doméstico. Os frutos da bananeira Maçã são os mais procurados, devido às suas características próprias de sabor, no entanto, seu cultivo tornou-se limitado pelo Mal-do-Panamá (Fusarium oxysporum f. sp cubense). Segundo Moreira (1999), o mercado interno absorve quase toda a produção, apresentando o maior consumo per capita.

No Brasil Central, os plantios têm se expandido e passaram a ser feitos com as variedades Nanicão, Enxerto e Terra. Existem, também, plantios da variedade Pacovan, comercializada, principlamente, em Brasília (Moreira 1999). A distância entre as regiões tradicionalmente produtoras e o seu potencial consumidor estimula o cultivo na região do Cerrado. Nesta região, com a utilização de irrigação, verificou-se produtividade média de 76,8 $\mathrm{t} \mathrm{ha}^{-1}, 44,9 \mathrm{t} \mathrm{ha}^{-1} \mathrm{e}$ $53,7 \mathrm{t} \mathrm{ha}^{-1}$, para os genótipos Grande Naine, Prata e falsa FHIA 18, respectivamente (Silva 2003). A irrigação é uma técnica que pode ajudar o bananicultor a aumentar a produtividade e qualidade do produto. Entretanto, para que a produção seja rentável e ambientalmente correta, requer bom planejamento e aplicação de técnicas modernas no manejo do sistema (Agrianual 2009).

No mercado de Goiânia, ocasionalmente, os genótipos Thap Maeo e Tropical são comercializados como sendo banana Maçã. Este fato prejudica a divulgação destes genótipos, uma vez que não é considerada a resistência à Sigatoka negra (Mycosphaerella fijiensis M.), em Thap Maeo, e a tolerância ao Mal-do-Panamá ( $F$. oxysporum f. sp cubense), em Thap Maeo e Tropical. Atualmente, a doença que mais preocupa as autoridades brasileiras é a Sigatoka negra, que impossibilita o transporte e a comercialização da produção para Estados livres da doença, sendo, também, o mais grave problema fitossanitário mundial para a produção de banana (Alves 1999).

Frente à necessidade de se resolverem problemas de fitossanidade, novos genótipos são pesquisados, passando pelos processos de melhoramento e difusão. Neste sentido, os genótipos Caipira, falsa FHIA 18, Thap Maeo e Tropical, e, segundo Cordeiro (2000), Grande Naine (grupo genômico AAA) e Prata (grupo AAB), que estão entre os mais difundidos, surgem como opção para os produtores. Entretanto, devem ser avaliadas as características físico-químicas e sensoriais de seus frutos, produzidos nas diferentes regiões de cultivo, e a utilização de tecnologias como a irrigação.

A utilização da irrigação passou a ser constante em bananais (Moreira 1999), pois, além de atender às necessidades das plantas, durante o momento de maior demanda, permite elevar a produção (Alves 1999). A região do Cerrado possui duas estações bem distintas, a seca e a chuvosa, ocorrendo nesta $80 \%$ do total da precipitação pluviométrica (Goedert 1989). Devido a estas características, a irrigação passou a ter uma maior importância para o desenvolvimento da cultura. Em busca de informações sobre a produtividade da bananicultura irrigada, nas condições edafoclimáticas da região, foram avaliadas, no presente estudo, as características produtivas dos genótipos falsa FHIA 18, Grande Naine, Prata e Thap Maeo, sob diferentes lâminas de irrigação, e a característica sensorial de sabor de frutos maduros dos quatro genótipos.

\section{MATERIAL E MÉTODOS}

O experimento foi realizado na área experimental da Escola de Agronomia e Engenharia de Alimentos da Universidade Federal de Goiás (EA/ UFG) $\left(16^{\circ} 36^{\prime} \mathrm{S}, 4^{\circ} 17^{\prime} \mathrm{W}\right.$ e altitude de $\left.730 \mathrm{~m}\right)$, em Goiânia (GO). O clima local, segundo a classificação de Köeppen, é do tipo Aw, ou seja, quente e semi-úmido, com estação seca bem definida. A precipitação média anual é de $1.576 \mathrm{~mm}$ e a insolação de 2.588 horas (Brasil 1992). O solo da área foi classificado como Latossolo Vermelho distroférrico.

$\mathrm{O}$ delineamento experimental utilizado foram blocos completos casualizados (DBC), em parcelas subdivididas, com quatro repetições. $\mathrm{O}$ fator de tratamento aplicado às parcelas correspondeu a quatro lâminas de irrigação $\left(\mathrm{L}_{1}\right.$ - testemunha: sem irrigação; $\mathrm{L}_{2}-40 \% ; \mathrm{L}_{3}-80 \%$; e $\mathrm{L}_{4}-120 \%$ ), expressas em percentagem da evapotranspiração potencial da cultura $(\mathrm{ETpc})$, determinada à partir da evaporação do tanque classe A, localizado a $500 \mathrm{~m}$ do experimento. A precipitação pluvial no período foi de $2.407,60 \mathrm{~mm}$ e as lâminas de água corresponderam a $\mathrm{L}_{1}-34,00 \mathrm{~mm}$; $\mathrm{L}_{2}-352,04 \mathrm{~mm} ; \mathrm{L}_{3}-704,08 \mathrm{~mm} ; \mathrm{e} \mathrm{L}_{4}-1.056,12 \mathrm{~mm}$.

Os genótipos falsa FHIA 18 (AAAB), Grande Naine (AAA), Prata e Thap Maeo (AAB) compreenderam o segundo fator de tratamento, aplicado às subparcelas. As parcelas experimentais, com as lâminas de irrigação, foram constituídas por três fileiras de 
10 plantas, em espaçamento $2,5 \mathrm{~m} \mathrm{x} \mathrm{1,6} \mathrm{m.} \mathrm{As} \mathrm{oito}$ plantas centrais, duas por genótipo, constituíram as parcelas úteis. Os tratos culturais foram realizados de acordo com recomendações de Alves (1999), do período de plantio das mudas, em 24 de janeiro de 2004, até o final do primeiro ciclo produtivo, em 11 de julho de 2005. As mudas utilizadas foram do tipo pedaço de rizoma, de, aproximadamente, $1,2 \mathrm{~kg}$.

O sistema de irrigação utilizado foi o de aspersão subcopa, com o uso de aspersores Naan 427 - AG (bocal com $3 \mathrm{~mm}$ de diâmetro). Em cada parcela, foram utilizados quatro aspersores, localizados na lateral da parcela, paralelos à fileira da cultura, no espaçamento de $8,50 \mathrm{~m}$, regulados em forma de setor, com ângulo de $180^{\circ}$, cobrindo toda a parcela. Para a caracterização físico-hídrica do solo, foram consideradas as camadas $0,0-0,2 \mathrm{~m}$ e $0,2-0,4 \mathrm{~m}$ de profundidade, realizando-se a determinação da densidade do solo segundo metodologia recomendada pela Embrapa (1997). As curvas de retenção de água no solo foram determinadas pelo método da centrífuga, conforme descrito por Freitas Júnior \& Silva (1984), nas tensões de água correspondentes a $6 \mathrm{kPa}$, $8 \mathrm{kPa}, 10 \mathrm{kPa}, 33 \mathrm{kPa}, 60 \mathrm{kPa}, 100 \mathrm{kPa}$ e $1.500 \mathrm{kPa}$ (Embrapa 1997), e o ajuste da curva foi realizado de acordo com metodologia proposta por Genuchten (1980), utilizando-se o software SWRC (Dourado Neto et al. 2000).

As umidades volumétricas na capacidade de campo $\left(\mathrm{q}_{\mathrm{CC}}\right)$ e ponto de murcha permanente $\left(\mathrm{q}_{\mathrm{PMP}}\right)$ foram obtidas empregando-se as curvas de retenção de água no solo, para as tensões de $6 \mathrm{kPa}$ e $1.500 \mathrm{kPa}$, respectivamente, sendo, na camada $0-0,2 \mathrm{~m}, \mathrm{q}_{\mathrm{CC}} \mathrm{e}$ $\mathrm{q}_{\text {PMP }}$ de $0,3487 \mathrm{~m}^{3} \mathrm{~m}^{-3}$ e $0,2551 \mathrm{~m}^{3} \mathrm{~m}^{-3}$, respectivamente, e, na camada $0,2-0,4 \mathrm{~m}$, de $0,3526 \mathrm{~m}^{3} \mathrm{~m}^{-3} \mathrm{e}$ $0,2773 \mathrm{~m}^{3} \mathrm{~m}^{-3}$. Desta forma, a capacidade de armazenamento de água, na camada $0-0,2 \mathrm{~m}$, foi de $18,7 \mathrm{~mm}$ e, na camada 0,2-0,4 m, de 15,1 mm. As irrigações foram realizadas quando a evapotranspiração potencial da cultura atingia um terço da capacidade de armazenamento de água no solo. O tempo de cada irrigação foi determinado pela razão entre a evapotranspiração potencial da cultura e a intensidade de aplicação de água, determinada em teste no campo.

A colheita dos cachos foi realizada quando os frutos apresentavam-se com calibre gordo (Alves 1999). Dos cachos colhidos, foram mensuradas as seguintes características: massa do cacho, da ráquis, das pencas e da segunda penca; número total de frutos, número de frutos da segunda penca, compri- mento do fruto e diâmetro do fruto central da fileira externa da segunda penca; comprimento do cacho; número de pencas; e número de frutos por penca. A partir da massa média das pencas, foi estimada a produção de frutos, em função da densidade de plantas (2.500 plantas ha $\left.{ }^{-1}\right)$. Os dados foram submetidos a análise de variância, através do aplicativo SAS (SAS Institute 1997), e as médias comparadas pelo teste $t$ de Student, a 5\%. Foram ajustadas equações de regressão para as características avaliadas que apresentaram efeito da aplicação das lâminas de irrigação. A partir das equações estimadas, elaboraram-se gráficos, com as respectivas curvas de resposta às lâminas e os respectivos coeficientes de determinação.

Para a caracterização sensorial dos genótipos falsa FHIA 18, Grande Naine, Prata e Thap Maeo, foram utilizadas 16 caixas de bananas, tipo torito, com capacidade de $18 \mathrm{~kg}$, colhidas em abril de 2005 . Cada caixa, forrada com filme de polipropileno, correspondia a uma lâmina e um genótipo, no entanto, para esta caracterização, foram considerados apenas os genótipos. No preenchimento das caixas, foram desprezadas as pencas das extremidades e, após este procedimento, as caixas foram levadas para a Central de Abastecimento de Goiás/SA (Ceasa-GO) e climatizadas em câmaras com temperatura de $18^{\circ} \mathrm{C}$, contendo gás etileno, por 48 horas, sendo, posteriormente, transportadas para a EA/UFG.

$\mathrm{Na}$ avaliação sensorial, foi utilizado o método afetivo de preferência, para o atributo sabor. A análise foi realizada no Laboratório de Análise Sensorial do Setor de Engenharia de Alimentos da EA/UFG. Os provadores, não treinados, foram recrutados entre os alunos, professores e funcionários da EA/UFG. As amostras dos frutos dos quatro genótipos, produzidas sob diferentes lâminas de irrigação, foram selecionadas, preparadas e oferecidas aos provadores, em cabines individuais e com iluminação adequada. $\mathrm{O}$ método sensorial afetivo de preferência, para o atributo sabor, foi realizado com 53, 50 e 44 provadores, respectivamente no $3^{\circ}, 4^{\circ}$ e $5^{\circ}$ dia de armazenamento após a climatização. $\mathrm{O}$ teste de preferência utilizado foi o de Comparação Múltipla (Chaves 2005) e o genótipo Prata foi utilizado como referência. Os provadores foram esclarecidos quanto ao preenchimento da ficha de avaliação (Chaves 2005). As amostras foram oferecidas ao acaso, em forma de fatias transversais dos frutos, com espessura de $1 \mathrm{~cm}$, juntamente com uma amostra-referência $\mathrm{R}$, em pratos descartáveis, identificados com números aleatórios de três dígitos. 
As intensidades das diferenças foram transformadas em escores de um a nove e, então, submetidas a análise de variância (teste F), e as médias referentes aos tratamentos comparadas pelo teste Tukey $(\mathrm{p}<0,05)$.

\section{RESULTADOS E DISCUSSÃO}

Com base nos resultados dos testes estatísticos, observou-se alta significância entre os genótipos, quando comparadas as variáveis estudadas.

Na caracterização dos cachos, durante a colheita do primeiro ciclo, verificou-se efeito significativo de lâminas de irrigação em todos os genótipos, sobre as variáveis massa do cacho, da ráquis e das pencas, e número total de frutos, comprimento do cacho e número de pencas.

Para o genótipo falsa FHIA 18, o efeito das lâminas de irrigação dentro de genótipos foi significativo na massa do cacho, massa das pencas, número


b total de frutos, comprimento do fruto, comprimento do cacho, número de pencas e número de frutos por penca, com resposta quadrática às lâminas de irrigação. Na característica avaliada de diâmetro do fruto central, houve efeito significativo das lâminas de irrigação, mas a regressão não se mostrou significativa para esta variável. Em Grande Naine, foi encontrada resposta quadrática na massa do cacho, massa da ráquis, massa das pencas, número total de frutos e número de pencas. O comprimento do cacho apresentou resposta linear. A resposta às lâminas de irrigação, no genótipo Thap Maeo, foi linear para massa do cacho e massa das pencas e quadrática para número total de frutos e número de pencas.

No genótipo falsa FHIA 18, foi observada resposta quadrática às lâminas de irrigação, na maioria das variáveis, na lâmina $\mathrm{L}_{4}$ deste genótipo. Esta tendência foi observada na massa do cacho, massa das pencas, número total de frutos (Figuras 1a e 1b),
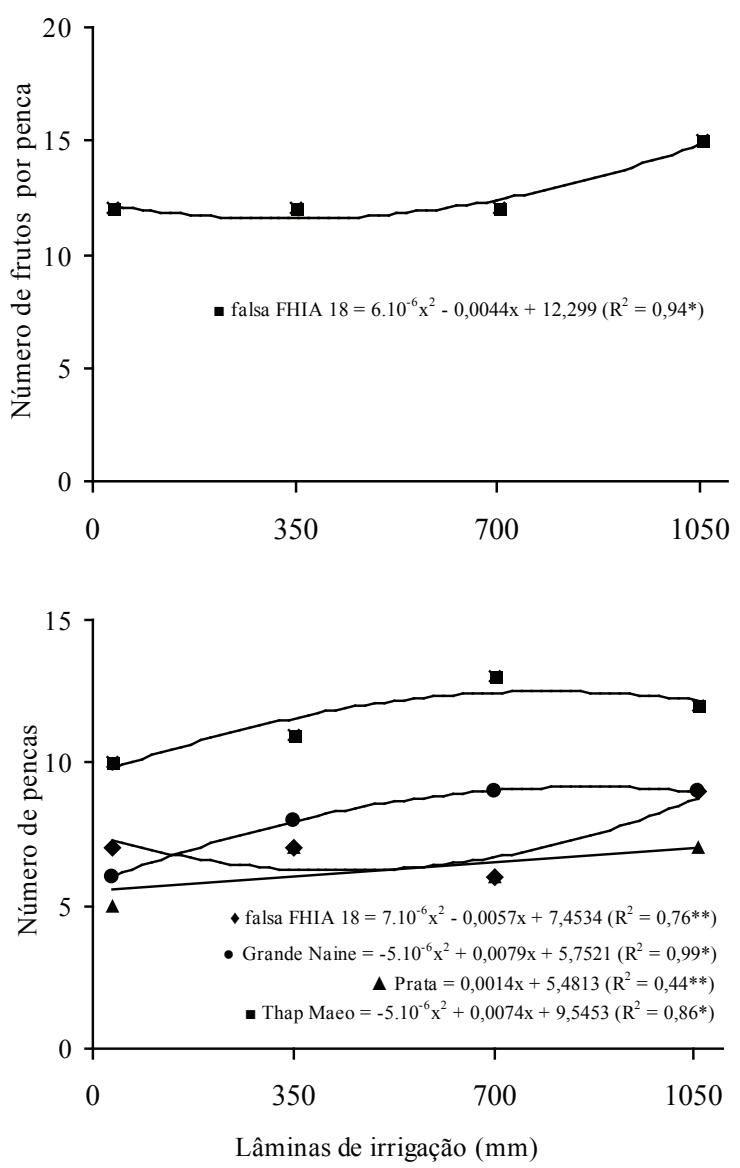

d

Figura 1. Massa das pencas dos genótipos falsa FHIA 18, Grande Naine e Thap Maeo (a); número total de frutos dos genótipos falsa FHIA 18, Grande Naine e Thap Maeo (b); número de frutos por penca do genótipo falsa FHIA 18 (c); e número de pencas dos genótipos falsa FHIA 18, Grande Naine, Prata e Thap Maeo (d), em função das lâminas de irrigação (Goiânia, GO, 2006). * e ** Valores significativos a $5 \%$ e $1 \%$, respectivamente. 
comprimento do fruto, comprimento do cacho e número de pencas (Figura 1d). O comportamento quadrático de Grande Naine foi inverso.

A influência das lâminas está relacionada aos componentes do cacho (que também influenciam na produção), dentre eles o número de frutos por penca, em falsa FHIA 18 (Figura 1c).

Os genótipos Grande Naine, Prata e Thap Maeo apresentaram valores médios para ciclo total de 475 dias e a falsa FHIA 18 para ciclo de 456 dias, que, apesar de menor, demonstra pequena diferença para os demais genótipos, de apenas 19 dias. Em estudos realizados por Braga Filho et al. (2008), os genótipos falsa FHIA 18, Grande Naine, Prata e Thap Maeo apresentaram ciclo vegetativo de 297, 344, 319 e 352 dias e ciclo produtivo de 157, 132, 155 e 121 dias, respectivamente. Santos et al. (2006) consideram que o ciclo é uma variável de fundamental importância para o melhoramento genético da bananeira, pois expressa sua precocidade. Em estudos realizados por Ramos et al. (2009), em Botucatu (SP), Grande Naine e Thap Maeo destacaram-se nos diferentes grupos genômicos, pois, dentre os mais produtivos, apresentaram menor ciclo e produtividade $\left(46,9 \mathrm{tha}^{-1}\right.$ e 25,8 $\mathrm{t} \mathrm{ha}^{-1}$, respectivamente). Estes dados são interessantes, pois Braga Filho et al. (2008) encontraram, na região do Cerrado, menores valores médios para o ciclo total dos dois genótipos. Quanto à produtividade, verificou-se, neste trabalho, maiores valores médios (Tabela 1).

Os genótipos falsa FHIA 18, Grande Naine, Prata e Thap Maeo apresentaram, em média, massa dos cachos de 12,64 kg; 23,65 kg; 12,64 kg; e $23,28 \mathrm{~kg}$, respectivamente (Tabela 1). A produtividade média estimada foi de $54,34 \mathrm{tha}^{-1}$, em Thap Maeo; 54,90 tha ${ }^{-1}$, em Grande Naine; 28,78 tha-1 ${ }^{-1}$ em falsa FHIA 18; e 28,88 tha-1 ${ }^{-1}$ no genótipo Prata. No entanto, estes valores são $46 \%, 28 \%$ e $36 \%$ inferiores, respectivamente, aos encontrados em falsa FHIA 18, Grande Naine e Prata, na mesma região, com o plantio realizado no mês de maio, empregando-se irrigação por gotejamento (Silva 2003). Considerando-se os valores médios por cultivar e lâminas de irrigação, as maiores médias, para a variável massa do cacho, foram obtidas para os genótipos falsa FHIA 18 e Prata, na $\mathrm{L}_{4}$, com 19,00 kg e 14,85 kg, e para Grande Naine e Thap Maeo, na $\mathrm{L}_{3}$, com 29,61 kg e 26,35 kg, respectivamente (Tabela 1). Isto mostra que, para se obterem altas produtividades, nas condições do Cerrado, deve-se utilizar suplementação hídrica ade- quada. Embora a comparação entre os genótipos não tenha sido o foco deste trabalho, suas médias, para as variáveis avaliadas, são apresentadas na Tabela 1 .

Os descritores massa do cacho e das pencas, embora expressem diretamente a produtividade, não podem ser considerados isoladamente na escolha de uma cultivar, pois outros caracteres relacionados aos frutos devem ser avaliados, como a massa, comprimento, diâmetro, sabor e resistência ao despencamento (Santos et al. 2006).

O número total de frutos por cacho foi inferior nas quatro lâminas, em falsa FHIA 18; mostrou-se superior nas lâminas $\mathrm{L}_{3}$ e $\mathrm{L}_{4}$, em Grande Naine; e superior em todas as lâminas, quando comparados aos obtidos por Silva et al. (2002), no primeiro ciclo. O número total de frutos e a massa do cacho de Thap Maeo, na ausência de irrigação $\left(\mathrm{L}_{1}\right)$, é relevante, pois foram $9 \%$ e $43 \%$ superiores, respectivamente, na região do Cerrado, quando comparados aos encontrados no Recôncavo Baiano, no primeiro ciclo (Silva et al. 2002). Silva (2003) verificou, em falsa FHIA 18 e Prata, a influência da irrigação no comprimento do fruto. O primeiro genótipo apresentou função quadrática e o segundo crescimento com equação linear. O número de frutos por penca destes genótipos não foi influenciado.

A resposta à irrigação do genótipo Prata, com crescimento linear, ocorreu somente no número de pencas (Figura 1d), sendo observadas, em média, cinco pencas na $L_{1}$, sete na $L_{2}$, seis na $L_{3}$ e sete na $L_{4}$ (Tabela 1). A bananeira Prata é vigorosa, conseguindo produzir em solos pobres e com deficiência hídrica, entretanto, apresenta baixa produtividade, mesmo em condições irrigadas, chegando a atingir 20-25 $\mathrm{t} \mathrm{ha}^{-1}$ ciclo $^{-1}$ (Alves 1999). Neste trabalho, foram alcançadas, na $\mathrm{L}_{2}, 30,49 \mathrm{t} \mathrm{ha}^{-1}$ (Tabela 1), no primeiro ciclo.

A massa e o número de frutos da segunda penca não responderam às lâminas de irrigação em nenhum dos genótipos. No entanto, o efeito de genótipos apresentou alta significância para as duas variáveis, devido às suas próprias características, quanto ao potencial de produção.

Os genótipos falsa FHIA 18 e Thap Maeo destacaram-se pela produção na região do Cerrado, e Thap Maeo pela resposta à irrigação e desempenho produtivo na ausência da irrigação. Os resultados obtidos por Santos et al. (2006) qualificam falsa FHIA 18 e Thap Maeo como promissoras para a região.

A maior produtividade estimada, verificada em falsa FHIA 18 e Grande Naine, foi de 43,69 tha-1 e 
Tabela 1. Massa do cacho (MC), massa da ráquis (MR), massa das pencas (MP), produtividade estimada $\left(\mathrm{P}_{\mathrm{e}}\right)$, número total de frutos $(\mathrm{NF})$, massa da segunda penca $\left(\mathrm{M}_{2} \mathrm{P}\right)$, número de frutos da segunda penca $\left(\mathrm{NF}_{2} \mathrm{P}\right)$, diâmetro do fruto $(\mathrm{DF})$, comprimento do fruto (CF), comprimento do cacho (CC), número de pencas (NP) e número de frutos por penca (NFP) dos genótipos de bananeira falsa FHIA 18, Grande Naine, Prata e Thap Maeo, em função das lâminas de irrigação (Goiânia, GO, 2006).

\begin{tabular}{|c|c|c|c|c|c|}
\hline \multirow{2}{*}{ Variáveis } & \multirow{2}{*}{$\begin{array}{l}\text { Lâminas } \\
\text { de irrigação }\end{array}$} & \multicolumn{4}{|c|}{ Genótipos } \\
\hline & & Falsa FHIA 18 & Grande Naine & Prata & Thap Maeo \\
\hline & $\mathrm{L}_{1}$ & $12,30 a b^{3}$ & $15,50 \mathrm{bc}$ & $9,81 \mathrm{a}$ & $18,30 \mathrm{c}$ \\
\hline $\mathrm{MC}$ & $\mathrm{L}_{2}$ & $10,33 \mathrm{a}$ & $24,01 \mathrm{~b}$ & $13,32 \mathrm{a}$ & $23,48 \mathrm{~b}$ \\
\hline \multirow{2}{*}{$(\mathrm{kg})$} & $\mathrm{L}_{3}^{2}$ & $8,92 \mathrm{a}$ & $29,61 \mathrm{~b}$ & $12,65 \mathrm{a}$ & $26,35 \mathrm{~b}$ \\
\hline & $\mathrm{L}_{4}$ & $19,00 \mathrm{a}$ & $25,47 \mathrm{~b}$ & $14,85 \mathrm{a}$ & $25,00 \mathrm{~b}$ \\
\hline CV (\%) & \multicolumn{5}{|c|}{30,97} \\
\hline \multirow{4}{*}{$\begin{array}{l}\text { MR } \\
(\mathrm{kg})\end{array}$} & $\mathrm{L}_{1}$ & $1,21 \mathrm{a}$ & $1,20 \mathrm{a}$ & $0,93 \mathrm{a}$ & $1,48 \mathrm{a}$ \\
\hline & $\mathrm{L}_{2}$ & $1,00 \mathrm{a}$ & $1,57 \mathrm{a}$ & $1,13 \mathrm{a}$ & $1,41 \mathrm{a}$ \\
\hline & $\mathrm{L}_{3}^{2}$ & $0,77 \mathrm{a}$ & $2,24 \mathrm{~b}$ & $1,05 \mathrm{a}$ & $1,73 \mathrm{~b}$ \\
\hline & $\mathrm{L}_{4}^{3}$ & $1,53 \mathrm{a}$ & $1,74 \mathrm{a}$ & $1,32 \mathrm{a}$ & $1,66 \mathrm{a}$ \\
\hline CV (\%) & \multicolumn{5}{|c|}{40,35} \\
\hline \multirow{4}{*}{$\begin{array}{l}\text { MP } \\
(\mathrm{kg})\end{array}$} & $\mathrm{L}_{1}$ & $11,09 \mathrm{ab}$ & $14,30 \mathrm{bc}$ & $8,87 \mathrm{a}$ & $16,82 \mathrm{c}$ \\
\hline & $\mathrm{L}_{2}$ & $9,33 \mathrm{a}$ & $22,44 \mathrm{~b}$ & $12,19 \mathrm{a}$ & $22,08 \mathrm{~b}$ \\
\hline & $\mathrm{L}_{3}^{2}$ & $8,15 \mathrm{a}$ & $27,37 \mathrm{~b}$ & $11,60 \mathrm{a}$ & $24,62 \mathrm{~b}$ \\
\hline & $\mathrm{L}_{4}^{3}$ & $17,47 \mathrm{a}$ & $23,73 \mathrm{~b}$ & $13,54 \mathrm{a}$ & $23,42 \mathrm{~b}$ \\
\hline CV (\%) & \multicolumn{5}{|c|}{30,51} \\
\hline \multirow{4}{*}{$\begin{array}{l}\mathrm{P}_{\mathrm{e}}{ }^{-} \\
\left(\mathrm{t} \mathrm{ha}^{-1}\right)\end{array}$} & $\mathrm{L}_{1}$ & $27,73 \mathrm{ab}$ & $35,75 \mathrm{bc}$ & $22,19 a$ & $42,04 \mathrm{c}$ \\
\hline & $\mathrm{L}_{2}$ & $23,33 \mathrm{a}$ & $56,10 \mathrm{~b}$ & $30,49 \mathrm{a}$ & $55,19 \mathrm{~b}$ \\
\hline & $\mathrm{L}_{3}^{2}$ & $20,36 \mathrm{a}$ & $68,43 \mathrm{~b}$ & $29,00 \mathrm{a}$ & $61,54 \mathrm{~b}$ \\
\hline & $\mathrm{L}_{4}^{3}$ & $43,69 \mathrm{a}$ & $59,33 \mathrm{~b}$ & $33,85 \mathrm{a}$ & $58,55 \mathrm{~b}$ \\
\hline $\mathrm{CV}(\%)$ & \multicolumn{5}{|c|}{30,51} \\
\hline \multirow{4}{*}{$\begin{array}{l}\mathrm{NF} \\
\left(\mathrm{n}^{\circ}\right)\end{array}$} & $\mathrm{L}_{1}$ & $81 \mathrm{~b}$ & $80 \mathrm{~b}$ & $55 \mathrm{a}$ & $162 \mathrm{c}$ \\
\hline & $\mathrm{L}_{2}^{1}$ & $82 \mathrm{a}$ & $116 \mathrm{~b}$ & $74 a$ & $184 \mathrm{c}$ \\
\hline & $\mathrm{L}_{3}^{2}$ & $75 a$ & $130 \mathrm{~b}$ & $78 \mathrm{a}$ & $205 \mathrm{c}$ \\
\hline & $\mathrm{L}_{4}^{3}$ & $125 \mathrm{~b}$ & $132 \mathrm{~b}$ & $82 \mathrm{a}$ & $199 \mathrm{c}$ \\
\hline $\mathrm{CV}(\%)$ & \multicolumn{5}{|c|}{17,93} \\
\hline \multirow{4}{*}{$\begin{array}{l}\mathrm{M}_{2} \mathrm{P} \\
(\mathrm{kg})\end{array}$} & $\mathrm{L}_{1}$ & $1,89 \mathrm{a}$ & $3,12 \mathrm{~b}$ & $1,81 \mathrm{a}$ & $2,13 \mathrm{a}$ \\
\hline & $\mathrm{L}_{2}$ & $1,64 \mathrm{a}$ & $3,66 \mathrm{~b}$ & $2,26 \mathrm{a}$ & $2,28 \mathrm{a}$ \\
\hline & $\mathrm{L}_{3}^{2}$ & $1,34 \mathrm{a}$ & $3,96 \mathrm{c}$ & $2,04 \mathrm{a}$ & $3,01 \mathrm{~b}$ \\
\hline & $\mathrm{L}_{4}^{3}$ & $2,37 \mathrm{a}$ & $3,24 \mathrm{~b}$ & $2,37 \mathrm{a}$ & $2,56 \mathrm{ab}$ \\
\hline CV (\%) & \multicolumn{5}{|c|}{31,02} \\
\hline & $\mathrm{L}_{1}$ & $14 \mathrm{ab}$ & $16 \mathrm{~b}$ & $11 \mathrm{a}$ & $19 \mathrm{c}$ \\
\hline & $\mathrm{L}_{2}^{1}$ & $15 \mathrm{ab}$ & $18 \mathrm{bc}$ & $12 \mathrm{a}$ & $19 \mathrm{c}$ \\
\hline$\left(n^{\circ}\right)^{2^{-}}$ & $\mathrm{L}_{3}^{2}$ & $13 \mathrm{a}$ & $17 \mathrm{a}$ & $13 \mathrm{a}$ & $21 \mathrm{~b}$ \\
\hline & $\mathrm{L}_{4}^{3}$ & $15 \mathrm{a}$ & $17 \mathrm{a}$ & $14 \mathrm{a}$ & $18 \mathrm{~b}$ \\
\hline CV (\%) & & & 22,65 & & \\
\hline & $\mathrm{L}_{1}$ & $33,37 \mathrm{a}$ & $41,44 \mathrm{~b}$ & 38,47 bc & $37,54 \mathrm{c}$ \\
\hline DF & $\mathrm{L}_{2}$ & $38,19 \mathrm{a}$ & $40,35 \mathrm{a}$ & $39,14 \mathrm{a}$ & $38,57 \mathrm{a}$ \\
\hline$(\mathrm{mm})$ & $\mathrm{L}_{3}^{2}$ & $35,22 \mathrm{a}$ & $40,12 \mathrm{bc}$ & $37,90 \mathrm{ab}$ & $41,72 \mathrm{c}$ \\
\hline & $\mathrm{L}_{4}^{3}$ & $37,10 \mathrm{a}$ & $38,08 \mathrm{a}$ & $39,39 \mathrm{a}$ & $40,04 \mathrm{a}$ \\
\hline CV (\%) & & & 8,76 & & \\
\hline & $\mathrm{L}_{1}$ & $18,47 \mathrm{ab}$ & $23,37 \mathrm{c}$ & $19,05 \mathrm{~b}$ & $16,26 \mathrm{a}$ \\
\hline $\mathrm{CF}$ & $\mathrm{L}_{2}$ & $16,66 \mathrm{a}$ & $23,49 \mathrm{~b}$ & $21,12 \mathrm{~b}$ & $16,42 \mathrm{a}$ \\
\hline$(\mathrm{cm})$ & $\mathrm{L}_{3}^{2}$ & $16,21 \mathrm{a}$ & $25,91 \mathrm{c}$ & $20,97 \mathrm{~b}$ & $16,81 \mathrm{a}$ \\
\hline & $\mathrm{L}_{4}^{3}$ & $20,16 \mathrm{ab}$ & $24,02 \mathrm{c}$ & $20,87 \mathrm{~b}$ & $17,69 \mathrm{a}$ \\
\hline CV (\%) & & & 12,68 & & \\
\hline & $\mathrm{L}_{1}$ & $32,17 \mathrm{a}$ & $41,19 \mathrm{~b}$ & $28,06 \mathrm{a}$ & $47,87 \mathrm{~b}$ \\
\hline $\mathrm{CC}$ & $\mathrm{L}_{2}$ & $26,56 \mathrm{a}$ & $51,22 \mathrm{c}$ & $36,56 \mathrm{~b}$ & $49,00 \mathrm{c}$ \\
\hline$(\mathrm{cm})$ & $\mathrm{L}_{3}^{2}$ & $21,75 \mathrm{a}$ & $58,06 \mathrm{c}$ & $34,37 \mathrm{~b}$ & $57,62 \mathrm{c}$ \\
\hline & $\mathrm{L}_{4}^{3}$ & $36,52 \mathrm{a}$ & $59,75 \mathrm{~b}$ & $38,75 \mathrm{a}$ & $53,06 \mathrm{~b}$ \\
\hline CV (\%) & & & 19,82 & & \\
\hline & $\mathrm{L}_{1}$ & $7 \mathrm{~b}$ & $6 \mathrm{ab}$ & $5 \mathrm{a}$ & $10 \mathrm{c}$ \\
\hline NP & $\mathrm{L}_{2}$ & $7 \mathrm{a}$ & $8 \mathrm{a}$ & $7 \mathrm{a}$ & $11 \mathrm{~b}$ \\
\hline$\left(n^{\circ}\right)$ & $\mathrm{L}_{3}^{2}$ & $6 a$ & $9 \mathrm{~b}$ & $6 a$ & $13 \mathrm{c}$ \\
\hline & $\mathrm{L}_{4}^{3}$ & $9 \mathrm{~b}$ & $9 \mathrm{~b}$ & $7 \mathrm{a}$ & $12 \mathrm{c}$ \\
\hline CV (\%) & & & 14,76 & & \\
\hline & $\mathrm{L}_{1}$ & $12 \mathrm{ab}$ & $13 \mathrm{~b}$ & $11 \mathrm{a}$ & $16 \mathrm{~b}$ \\
\hline NFP & $\mathrm{L}_{2}$ & $12 \mathrm{a}$ & $15 \mathrm{~b}$ & $11 \mathrm{a}$ & $16 \mathrm{~b}$ \\
\hline$\left(n^{\circ}\right)$ & $\mathrm{L}_{3}^{2}$ & $12 \mathrm{a}$ & $14 \mathrm{~b}$ & $12 \mathrm{a}$ & $16 \mathrm{~b}$ \\
\hline & $\mathrm{L}_{4}^{3}$ & $15 \mathrm{~b}$ & $15 \mathrm{~b}$ & $12 \mathrm{a}$ & $17 \mathrm{c}$ \\
\hline CV (\%) & & & 11,45 & & \\
\hline
\end{tabular}

${ }^{1}$ Médias seguidas pela mesma letra na linha, entre cultivares, não diferem entre si, pela estatística t de Student, a 5\%. 
$68,43 \mathrm{t} \mathrm{ha}^{-1}$, nas lâminas $\mathrm{L}_{4}$ e $\mathrm{L}_{3}$, respectivamente. No entanto, o incremento da produção, com o uso destes níveis de irrigação, deve ser avaliado quanto à viabilidade econômica e retorno do investimento. No primeiro nível de irrigação estabelecido $\left(\mathrm{L}_{2}\right)$, foi obtido incremento na produção de $37 \%$ (Prata) e $31 \%$ (Thap Maeo), em relação à $\mathrm{L}_{1}$. A produção não se alterou nas lâminas $\mathrm{L}_{3}$ e $\mathrm{L}_{4}$ destes dois genótipos.

$\mathrm{Na}$ análise sensorial de preferência, os valores médios das notas dos provadores, no terceiro dia de armazenamento, não indicaram diferença de preferência dos genótipos de banana avaliados, em relação ao Prata $(p>0,05)$, quanto ao sabor. Contudo, no quarto e quinto dias de armazenamento, foram observadas diferenças significativas $(\mathrm{p} \leq 0,05)$ entre os diferentes genótipos, quanto à preferência dos provadores. No quarto dia, as bananas Grande Naine e falsa FHIA 18 foram tão preferidas quanto a Prata, sendo a Thap Maeo menos preferida. No quinto dia de armazenamento, apenas o genótipo Grande Naine permaneceu tão preferido quanto o de banana Prata. Nas condições em que foram realizadas as análises, pode-se considerar que o sabor da banana Grande Naine foi o que mais se assemelhou ao da banana Prata, durante o período de avaliação (Tabela 2).

A escolha da banana Prata, como amostra-referência, pode ter influenciado na maior preferência de falsa FHIA 18, no quarto dia de avaliação, em detrimento de Thap Maeo, uma vez que a falsa FHIA 18 é um híbrido de Prata (Cordeiro 2000). Matsuura et al. (2002) destacaram a importância dos atributos sabor e cor, para a análise sensorial de frutos de bananeira, relacionando o sabor à preferência pelo genótipo e a cor à atratividade do consumidor. Para Damatto Júnior et al. (2005), o sabor típico de Prata justificou a preferência do consumidor pelo genótipo

Tabela 2. Médias de notas de preferência dos julgadores, para as amostras de cada genótipo de banana, no terceiro, quarto e quinto dias de armazenamento após a climatização (Goiânia, GO, 2006).

\begin{tabular}{lccc}
\hline \multirow{2}{*}{ Genótipo } & \multicolumn{3}{c}{ Notas dos julgadores $^{2}$ no período de armazenamento } \\
\cline { 2 - 4 } & $3^{\circ}$ dia & $4^{\circ}$ dia & $5^{\circ}$ dia \\
\hline Falsa FHIA 18 & $4,66 \mathrm{a}$ & $4,56 \mathrm{ab}$ & $3,52 \mathrm{~b}$ \\
Grande Naine & $4,55 \mathrm{a}$ & $4,80 \mathrm{a}$ & $4,32 \mathrm{ab}$ \\
Prata & $4,49 \mathrm{a}$ & $4,76 \mathrm{a}$ & $4,70 \mathrm{a}$ \\
Thap Maeo & $4,09 \mathrm{a}$ & $3,76 \mathrm{~b}$ & $3,50 \mathrm{~b}$ \\
\hline Médias & 4,45 & 4,47 & 4,01 \\
\hline CV $(\%)$ & 46,29 & 41,12 & 44,39 \\
\hline
\end{tabular}

${ }^{1}$ Médias seguidas por letras distintas, na coluna, diferem pelo teste Tukey, a $5 \%$.
Prata Anã, em detrimento de Prata Zulu. Este fato confirma a importância da avaliação dos atributos relacionados à qualidade de frutos, principalmente $\mathrm{o}$ sabor, em programas de melhoramento e difusão de genótipos (Matsuura et al. 2002, 2004).

Devido às características próprias da região do Cerrado, o uso da irrigação torna-se relevante para suprir a necessidade hídrica da bananeira, em determinadas fases do seu crescimento e desenvolvimento, quando ocorridas no período seco. Para tanto, a atividade produtiva deve ter como alvo o mercado consumidor, que apresenta informações determinantes para o nível de investimento a ser empregado. O uso de novos genótipos tornou-se importante com o aparecimento de problemas fitossanitários, principalmente a Sigatoka negra. Desta forma, falsa FHIA 18 e Thap Maeo, que são resistentes, podem não vencer o conservadorismo do mercado varejista, em um primeiro momento, mas surgem como opção para cultivo em pomares domésticos e de pequenas propriedades rurais.

Na introdução de genótipos resistentes ou tolerantes a pragas e doenças nocivas à cultura, sugerem-se ações mais agressivas de marketing, por parte de instituições ligadas ao setor, como a degustação em supermercados, feiras livres e no comércio em geral. Silva et al. (2002) mencionam que o sabor é um dos atributos relacionados ao fruto que mais influenciam na preferência do consumidor. Portanto, é necessário um melhor estudo da curva de preferência de cada cultivar, de forma que seus frutos possam ser apresentados aos provadores em uma faixa considerada mais adequada para o consumo. Tem-se observado que alguns genótipos expressam melhor seu potencial quando bem maduros. Este fato é interessante sob a ótica de um maior período de prateleira do genótipo. No entanto, esta característica necessita ser do conhecimento do consumidor, para que este aumente seu interesse pelo genótipo, principalmente no caso dos genótipos desconhecidos.

\section{CONCLUSÕES}

1. Os genótipos falsa FHIA 18, Grande Naine e Thap Maeo são responsivos à irrigação.

2. A maior produtividade estimada para Grande Naine e Thap Maeo foi encontrada na $\mathrm{L}_{3}$ e, para falsa FHIA 18 e Prata, na $\mathrm{L}_{4}$.

3. Thap Maeo é uma promissora variedade para a região do Cerrado goiano, com boa produção, mesmo sem irrigação. 
4. Os genótipos Prata e Grande Naine são os preferidos dos consumidores, embora Thap Maeo e FHIA 18 apresentem potencial para aproveitamento local e regional.

\section{AGRADECIMENTOS}

Ao Conselho Nacional de Desenvolvimento Científico e Tecnológico (CNPq), pela concessão de bolsa de doutorado, ao primeiro autor, e bolsa de iniciação científica, ao último autor.

\section{REFERÊNCIAS}

AGRIANUAL: anuário da agricultura brasileira. São Paulo: FNP Consultoria \& Comércio, 2009.

ALVES, E. J. A cultura da banana: aspectos técnicos, socioeconômicos e agroindustriais. 2. ed. Brasília, DF: Embrapa, 1999.

BRAGAFILHO, J. R. et al. Crescimento e desenvolvimento de cultivares de bananeira irrigadas. Revista Brasileira de Fruticultura, Jaboticabal, v. 30, n. 4, p. 981-988, dez. 2008.

BRASIL. Ministério da Agricultura e Reforma Agrária. Secretaria Nacional de Irrigação. Departamento Nacional de Meteorologia. Normais climatológicas: 1961-1990. Brasília, DF: DNMet, 1992.

CHAVES, J. B. P. Métodos de diferença em avaliação sensorial de alimentos e bebidas. Viçosa: UFV, 2005.

CORDEIRO, Z. J. M. (Org.). Banana: produção: aspectos técnicos. Brasília, DF: Embrapa, 2000.

DAMATTO JÚNIOR, E. R. et al. Produção e caracterização de frutos de bananeira 'Prata-Anã' e 'Prata-Zulu'. Revista Brasileira de Fruticultura, Jaboticabal, v. 27, n. 3, p. 440443, dez. 2005.

DOURADO NETO, D. et al. Software to model soil water retention curves (SWRC, version 2.0). Scientia Agricola, Piracicaba, v. 57, n.1, p. 191-192, 2000.

EMPRESA BRASILEIRA DE PESQUISA AGROPECUÁRIA (Embrapa). Manual de métodos de análise de solo. Rio de Janeiro: Embrapa/CNPS, 1997.

FREITAS JÚNIOR, E.; SILVA, E. M. Uso da centrífuga para determinação da curva de retenção de água no solo, em uma única operação. Pesquisa Agropecuária Brasileira, Brasília, DF, v. 19, n. 11, p. 1423-1428, nov. 1984.

GENUCHTEN, M. V. A closed-form equation for predicting the hydraulic conductivity of unsaturated soils.
Soil Science American Journal, Madison, v. 44, n. 5, p. 892-898, 1980.

GOEDERT, W. J. Região dos cerrados: potencial agrícola e política para seu desenvolvimento. Pesquisa Agropecuária Brasileira, Brasília, DF, v. 24, n. 1, p. 1-17, 1989.

MATSUURA, F. C. A. U.; CARDOSO, R. L.; RIBEIRO, D. E. Qualidade sensorial de frutos de híbridos de bananeira cultivar Pacovan. Revista Brasileira de Fruticultura, Jaboticabal, v. 24, n. 1, p. 263-266, abr. 2002.

MATSUURA, F. C. A. U.; COSTA, J. I. P.; FOLEGATTI, M. I. S. Marketing de banana: preferências do consumidor quanto aos atributos de qualidade dos frutos. Revista Brasileira de Fruticultura, Jaboticabal, v. 26, n. 1, p. 4852, abr. 2004.

MOREIRA, R. S. Banana: teoria e prática de cultivo. 2. ed. Campinas: Fundação Cargill, 1999.

RAMOS, D. P. et al. Avaliação de genótipos de bananeira em Botucatu - SP. Revista Brasileira de Fruticultura, Jaboticabal, v. 31, n. 4, p. 1092-1101, dez. 2009.

SANTOS, S. C. et al. Caracterização morfológica e avaliação de cultivares de bananeira resistentes à Sigatoka negra (Mycosphaerella fijiensis Morelet) no sudoeste goiano. Revista Brasileira de Fruticultura, Jaboticabal, v. 28, n. 3, p. 449-453, dez. 2006.

SAS INSTITUTE. SAS/Stat software: changes and enhancements through release 6.12. Cary: SAS Institute Inc., 1997.

SILVA, L. B. Crescimento, desenvolvimento e produtividade de cultivares de banana (Musa sp.) submetidas a diferentes lâminas de irrigação. 2003. 58 f. Dissertação (Mestrado em Produção Vegetal)-Escola de Agronomia e Engenharia de alimentos, Universidade Federal de Goiás, Goiânia, 2003.

SILVA, S. O.; FLORES, J. C. O.; LIMA NETO, F. P. Avaliação de cultivares e híbridos de bananeira em quatro ciclos de produção. Pesquisa Agropecuária Brasileira, Brasília, DF, v. 37, n. 11, p. 1567-1574, nov. 2002. 